\title{
Hotspots Detection in Photovoltaic Modules Using Infrared Thermography
}

\author{
April M. Salazar ${ }^{1,2}$ and Erees Queen B. Macabebe ${ }^{1}$ \\ ${ }^{1}$ Ateneo de Manila University, Department of Electronics, Computer, and Communications Engineering, Quezon City, Philippines \\ ${ }^{2}$ Ateneo de Davao University, ECE Department, School of Engineering and Architecture, Davao City, Philippines
}

\begin{abstract}
An increased interest on generating power from renewable sources has led to an increase in solar photovoltaic (PV) system installations worldwide. Power generation of such systems is affected by factors that can be identified early on through efficient monitoring techniques. This study developed a non-invasive technique that can detect localized heating and quantify the area of the hotspots, a potential cause of degradation in photovoltaic systems. This is done by the use of infrared thermography, a well-accepted non-destructive evaluation technique that allows contactless, real-time inspection. In this approach, thermal images or thermograms of an operating PV module were taken using an infrared camera. These thermograms were analyzed by a Hotspot Detection algorithm implemented in MATLAB. Prior to image processing, images were converted to CIE L*a*b color space making kmeans clustering implementation computationally efficient. K-means clustering is an iterative technique that segments data into $k$ clusters which was used to isolate hotspots. The devised algorithm detected hotspots in the modules being observed. In addition, average temperature and relative area is provided to quantify the hotspot. Various features and conditions leading to hotspots such as crack, junction box and shading were investigated in this study.
\end{abstract}

\section{Introduction}

There has been a rising awareness worldwide on the use of solar photovoltaic energy. Reasons behind are not only for addressing climate change, but also for generating new financial opportunities and most importantly for giving energy access to countless people still deprived of modern energy facilities. This global clamor to harness energy from the sun has grown rapidly over the past decade as stated in the Renewables 2015 Global Status Report which underscored that solar PV global capacity increased from 3.7 Gigawatts in 2004 to 177 Gigawatts in 2014. Of this, 40 gigawatts was added in 2014 alone [1].

Even in the Philippines, solar PV installations have grown rapidly as evidenced by the numerous projects implemented across the country including installations in shopping malls, residences and commercial establishments. The largest installation to date is the 100MW solar power plant in Cadiz City, Negros Occidental.

The concern now however is that, after these solar modules have been installed, less attention is given to evaluating whether or not these modules are operating at its optimal rated conditions after five or ten years of use. An ideal solar PV module is made to last for 20 to 25 years. However due to various factors, this lifespan is reduced and without proper monitoring, one will never know if a solar PV module has already become unproductive. Usually, the cause of these damages starts out as a hotspot.

A hotspot is a defect found in a solar photovoltaic module that negatively affects the normal operation of the PV module. There are several conditions that may lead to a hotspot. One simple cause is shading. Shading can be produced by a branch of a tree, an antenna, a nearby building, dirt or anything that physically blocks the solar PV module. What happens is that, when one cell is shaded, it reduces the current through the good cells. The good cells that produce higher voltages usually reverse bias the bad cell. When this occurs, there is large dissipation of power in the poor cell. Large dissipation of power in a small area results to local overheating or hotspots. This can lead to continual yield losses and in worst case scenarios, hotspots can even catch fire [2].

Another potential cause of hotspot is an existing defect in the module like a crack or a dent. This defect becomes a load thus current also concentrate to this area, causing an enormous dissipation of power in the damaged cell, hence, creating a hotspot. As a result, the output power decreases, thereby decreasing the efficiency of the PV module.

Current-voltage $(I-V)$ characterization is used to evaluate the performance of the solar PV modules and obtain parameters such as maximum power output and conversion efficiency. However, it is impractical to do in 
large-scale PV systems because modules need to be individually disconnected from the array and attached to the $I-V$ curve tracers. It is, therefore, time-consuming and inconvenient if large solar PV power plants are to be considered.

With the said considerations in mind, a thermal imaging system that can be used to evaluate the operating conditions of a solar PV module is introduced in this study. Thus, the objectives of this study are as follows: (1) Develop a non-invasive technique to locate hotspots in the solar PV module using an infrared camera and a hotspot detection algorithm, (2) Isolate areas with higher temperature intensities using k-means clustering and quantify these hotspots by determining the average temperature and identifying the relative size (area), and (3) Determine possible factors or conditions contributing to hotspots.

This technique eliminates the need to detach each solar PV module from the array for testing since it can identify damaged or problematic PV modules through the thermograms and relative size of the hotspots, which can then be verified through $I-V$ characterization.

\section{Background}

\subsection{Infrared thermography}

Amongst existing condition monitoring techniques and non-destructive testing methods today, infrared thermography is considered a promising tool for fast and reliable fault detection. Infrared thermography uses midwave (MWIR, from $\sim 3$ to $5 \mu \mathrm{m}$ ) or long-wave (LWIR, from $\sim 7$ to $14 \mu \mathrm{m}$ ) infrared sensors to obtain thermal images or thermograms of objects under inspection. Based on Planck's black body radiation law, all objects emit infrared radiation proportional to their temperatures. For instance, a study lamp which is turned off will emit low power radiation at wavelengths mostly higher than 1 $\mu \mathrm{m}$, outside the human visible range. If the lamp is turned on, the temperature will rise and it will glow red due to the higher energies emitted and enters the visible spectrum. If it continues to rise in temperature, radiation may change from red to violet and then the light looks white [3]. Hence, IR thermography is a feasible tool to determine the surface temperature as well as any abnormalities to the temperature trend of a body, or a solar PV module under inspection.

\subsection{K-means clustering}

In this study, k-means clustering algorithm is taken as a segmentation tool to proceed with the processing of the infrared images. K-Means algorithm categorizes the input data points into multiple groups based on their intrinsic distance from each other. The algorithm aims to find groupings among them by gathering around a centroid. The points are clustered around centroids, $k$, which is achieved by

$$
V=\sum_{i=1}^{k} \sum_{x_{j} \in S_{i}}\left(x_{j}-\mu_{i}\right)^{2}
$$

where $\mathrm{S}_{i}$ are the clusters created, $i=1,2, \ldots k$ and $\mu_{i}$ is the mean of all the points $x_{j} \in S_{i}$. [4]. The cycle of recomputing and assigning data points to the centroid repeats until the centroids do not change or when a certain threshold or criteria is achieved. The most important parameter to consider in this type of clustering is the value of $k$. It is important that $k$ is appropriate to the image being clustered. This is why some researchers prefer $k$ to be unsupervised based on the color patterns of the image under study [5].

\subsection{CIE $L{ }^{*} a{ }^{*} b^{*}$ color space}

Prior processing, the RGB values of the IR images were converted to CIE $L^{*} a * b^{*}$. CIELAB, like the RGB, is a color space, specified by the International Commission on Illumination (Commission internationale de l'éclairage, hence its CIE acronym). The three coordinates of CIELAB represent the lightness of the color, with $L^{*}=0$ yields black and $L^{*}=100$ is white. The $a^{*}$ represent its position between red and green, where negative values indicate green and positive values indicate red. Its position between yellow and blue is represented as $b^{*}$ where negative values indicate blue and positive values indicate yellow. Distinct from the RGB and CMYK color space, $L^{*} a^{*} b^{*}$ color is intended to approximate human vision and seeks perceptual uniformity. $L^{*}$ component also closely matches human interpretation of lightness [6].

\section{Experimental methods}

FLIR C2 infrared camera was utilized in this study. It is a portable infrared camera with an infrared sensor resolution of $80 \times 60$ and a thermal sensitivity of $<0.10^{\circ} \mathrm{C}$. The camera, having a range of $7.5-14 \mu \mathrm{m}$, can display the object temperature within the range of $-10^{\circ} \mathrm{C}$ to $+150^{\circ} \mathrm{C}$. Two camera setting conditions had to be considered when taking infrared images of the PV module under inspection. Emissivity $e$ is the ratio of the energy radiated from a material's surface to that radiated from a blackbody under the same temperature conditions and wavelength [7]. Objects normally have emissivity ranging from 0.1 to 0.95 . In this case, $e=0.90$ was used, within the typical value range for glass. Another setting condition is the reflective temperature which was set at $10^{\circ} \mathrm{C}$, as recommended by the camera manufacturer. This parameter compensates for the radiation reflected on the object and those between the camera and the PV module.

Distance setting between the camera and the PV module was set at $1 \mathrm{~m}$. Furthermore, the images should be taken at camera angle between $5^{\circ}$ and $60^{\circ}$ to avoid casting shadows on the PV module [8]. In this study, images were taken at angles between $20^{\circ}$ to $35^{\circ}$, estimated with respect to a line normal to the surface of the PV module. Several images were taken at several time intervals to eliminate the effects of glare or temporary shading factors like clouds in the data. With these settings, infrared images of the solar PV module were collected.

Irradiance, ambient temperature and module temperature were also recorded during the image 
collection stage. A temperature data logging circuit using DS18B20 digital temperature sensors and an Arduino Uno microcontroller was devised to measure the temperature of the PV module at four points. A DHT22 temperature and humidity sensor was used to measure the ambient temperature as well as the relative humidity of the environment. For the irradiance measurement, Daystar DS-05 Solar meter was used. For the image processing component of the system, the Image Processing Toolbox in MATLAB was utilized. Fig. 1 illustrates the hotspot detection process.

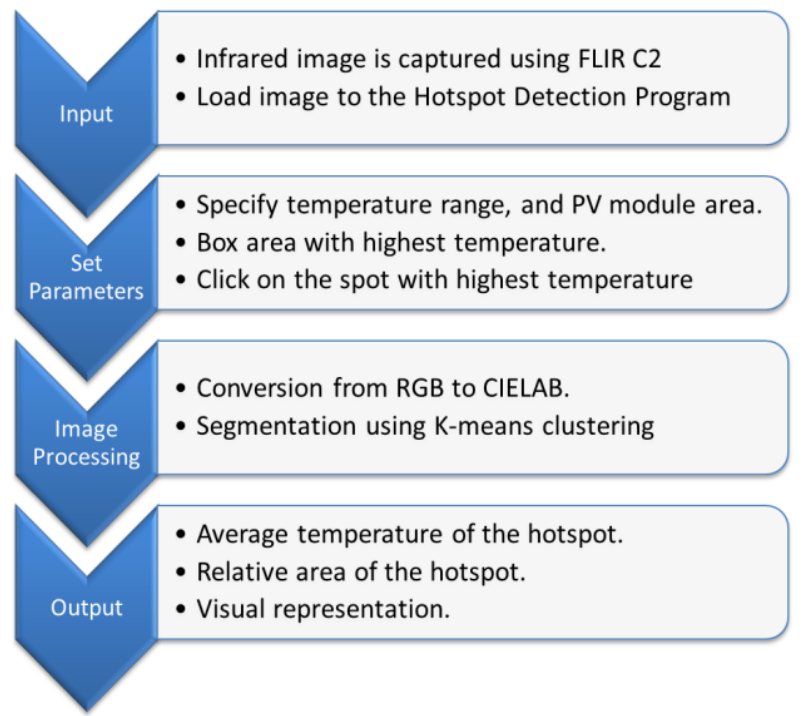

Figure 1. Hotspot detection flow

An infrared image of the PV module is obtained and loaded to the Hotspot Detection Program. The minimum and maximum temperature is then specified by the user for internal computations of the average temperature of the hotspot. These values are displayed in the color bar of the infrared image. The user also specifies the solar PV module area by clicking the four corners of the PV module. Due to camera limitations, it is possible that only parts of the module are captured and not the entire solar PV module. The relative percent area of the potential hotspot is computed based on the specified PV module area. To further specify the spot with the highest temperature, it is necessary to box in the area with the highest temperature.

To lessen the color into 2 dimensions, the RGB values of the image are converted to CIE $L^{*} a^{*} b^{*}$. The conversion from RGB to $\mathrm{CIE} L^{*} a^{*} b^{*}$ reduces the computational complexity of the algorithm since the color scale was reduced from 3 to 2 dimensions. This minimizes scattering or large variations of clusters and reduces computation time.

Image segmentation utilizes k-means clustering. Kmeans is used to group pixels that are in the same temperature range. Data points are grouped to the nearest cluster index using Euclidean distance. After the image is clustered into $k$ groups, the user must select the cluster with the highest temperature range then, finally, the isolated hotspot is displayed including the average temperature value and relative percent area affected by the hotspot.

\section{Results and discussions}

\subsection{Hotspots detection program}

Fig. 2 displays an implementation of the Hotspot Detection algorithm developed in the study with $k=3$. In this run, the potential hotspot has an area of $13.08 \%$ and a temperature of about $51{ }^{\circ} \mathrm{C}$. As seen in the figure, the Hotspot Detection algorithm developed was able to isolate the area with localized heating.
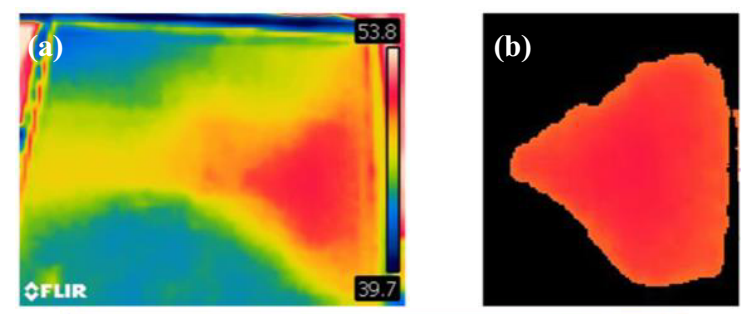

(c)

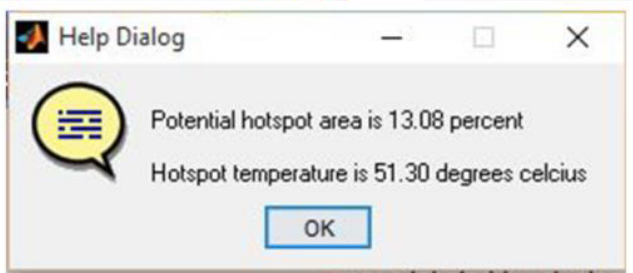

Figure 2. (a) Input Infrared Image (b) Isolated potential hotspot, (c) Summary of area and temperature of the hotspot

One PV module considered for the study is a polycrystalline silicon PV module rated at 50W. Fig. 3 shows a sample run on the IR image of the $50 \mathrm{~W}$ PV module using the hotspot detection algorithm developed.

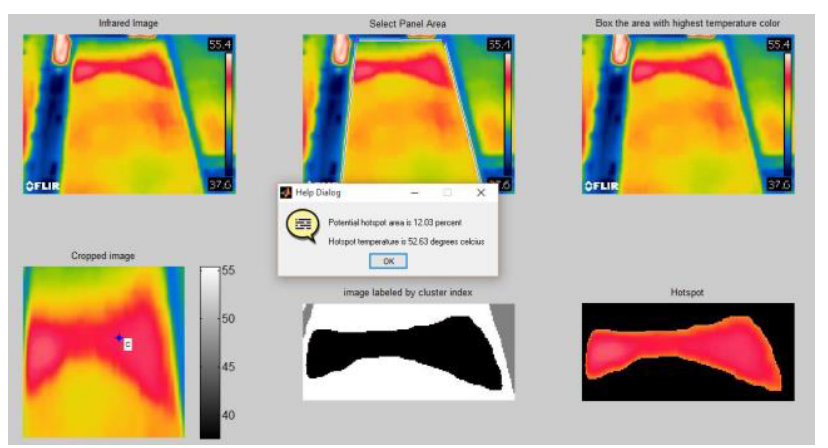

Figure 3. Hotspot on a $50 \mathrm{~W}$ solar PV module showing a relative area percentage of $12.03 \%$ at $52^{\circ} \mathrm{C}$, Irr $=749 \mathrm{~W} / \mathrm{m}^{2}$.

\subsection{Cracks cause hotspots}

The IR image of the 50W PV module in Fig. 3 show a relatively large hotspot. Due to its age, it is possible that PV module has accumulated defects but was not diagnosed since its deployment. Fig. 4 show that an area on the upper part of the 50W PV module consistently measured the highest temperature. Images were taken at different days to ensure that the hotspot was not caused by clouds or any other temporary obstruction that may cause shading. Despite changing the capture angle, as seen in Fig. 4 (c) and (d), a consistent white spot is observed. Using the algorithm on one of its IR image, this particular hotspot measured an average temperture of $52^{\circ} \mathrm{C}$. 

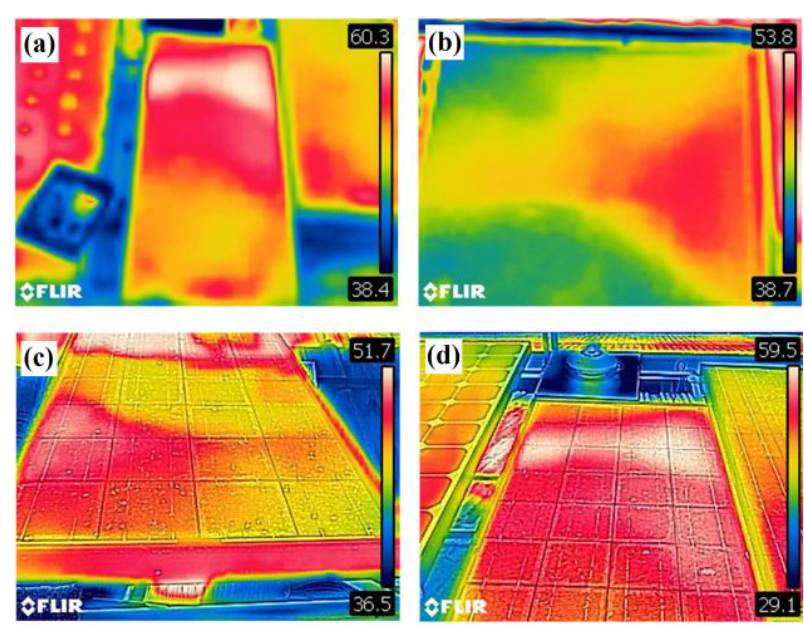

Figure 4. Infrared images of the $50 \mathrm{~W}$ Module taken as (a) whole module, (b) half module, (c) and (d) at different angles.

By inspecting the PV module, the area with the potential hotspot contains a crack. This defect caused this region to dissipate more power. Hence, produced colors corresponding to higher temperatures. To further validate this observation, temperature at varied points of the panel was recorded using the temperature data logging circuit created.

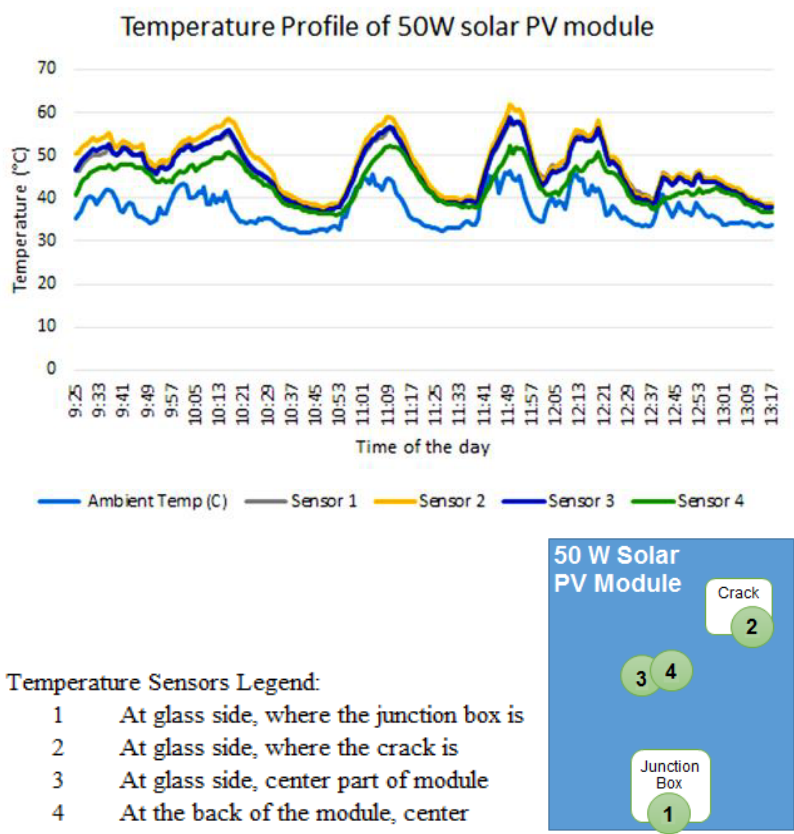

Figure 5. Temperature readings at 4 points of the panel

Sensor 2, placed on the cracked area of the PV module, has recorded higher temperature readings when compared with sensors 1 and 3 . Sensor 2 has an average temperature difference of $1.6^{\circ} \mathrm{C}$ to sensor 1 and $1.3^{\circ} \mathrm{C}$ to sensor 3 . In addition, sensor 2 has an average temperature difference of $4.5^{\circ} \mathrm{C}$ when compared to sensor 4 readings which is expected since sensor 4 is located at the back of the PV module. This supports the initial observation that the crack caused localized heating in the module.

\subsection{Junction box as a source of hotspots}

Common among the PV modules investigated in this study is that localized heating was observed in the areas where the junction box is located. Figure 6 shows IR images of another aged 50W polycrystalline PV module. It did not manifest any other hotspot aside from the area at the bottom of the image where the junction box is located. Using the Hotspot Detection algorithm, the hotspot was identified to be about $1.9 \%$ at around $54{ }^{\circ} \mathrm{C}$.
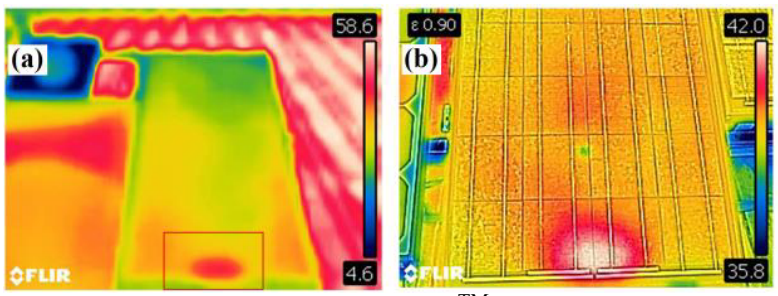

Figure 6. (a) Infrared and (b) MSX ${ }^{\mathrm{TM}}$ images of the $50 \mathrm{~W}$ solar PV module with junction box, indicated in (a), as cause of hotspot.

To confirm this observation, different solar PV modules were investigated. Fig. 7 presents the IR image of (a) a $10 \mathrm{~W}$ polycrystalline PV module, and two monocrystalline PV modules with peak power ratings at (b) $280 \mathrm{~W}$ and (c) $250 \mathrm{~W}$. They were found to exhibit the same condition, that the area where the junction box is located created thermal stress on the PV module and thus lead to hotspots. The junction box contains the bypass diodes of the solar module.

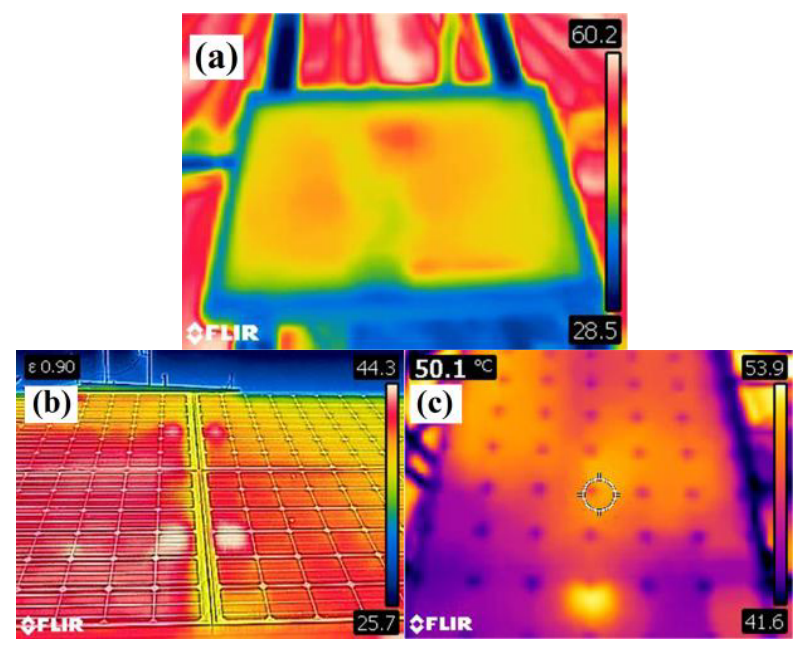

Figure 7. Different solar PV modules with junction box as source of hotspot: (a) $10 \mathrm{~W}$ Polycrystalline, (b) $280 \mathrm{~W}$ Monocrystalline, and (c) 250W Monocrystalline PV modules.

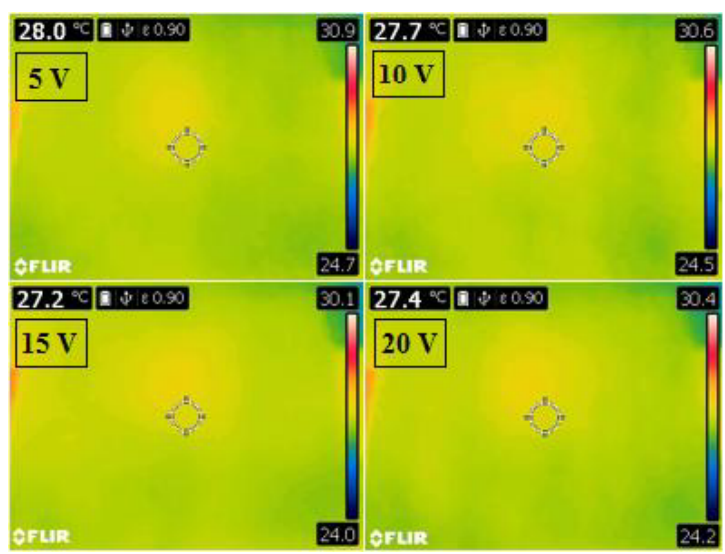

Figure 8. IR images of the $10 \mathrm{~W}$ PV module under reverse-bias conditions: $5 \mathrm{~V}, 10 \mathrm{~V}, 15 \mathrm{~V}$ and $20 \mathrm{~V}$. 


\subsection{Shading factors lead to hotspots}

A reverse-bias test was conducted on a $10 \mathrm{~W}$ polycrystalline silicon PV module. Under reverse-bias conditions, external current is fed to the solar PV module and the solar cells begin to emit light in the infrared range. Defects in the material fail to produce light and so faults become apparent. Therefore, it is possible to detect material defects using the reverse-bias test. Fig. 8 shows the IR images of the $10 \mathrm{~W}$ PV module under reverse-bias conditions where no defects were detected.

To confirm this defect-free condition, infrared images were taken with the module under operating conditions. Fig. 9 shows the IR images and the corresponding photos of the PV module taken under operating conditions. Initial infrared images, however, yielded a particular hotspot pattern shown in Fig. 9(a). There were observable red spots on the PV module that were not evident during the reverse-bias test. Upon checking, the glass surface of the PV module contained adhesive remains, the patterns of which correspond to the pattern of the red spots.
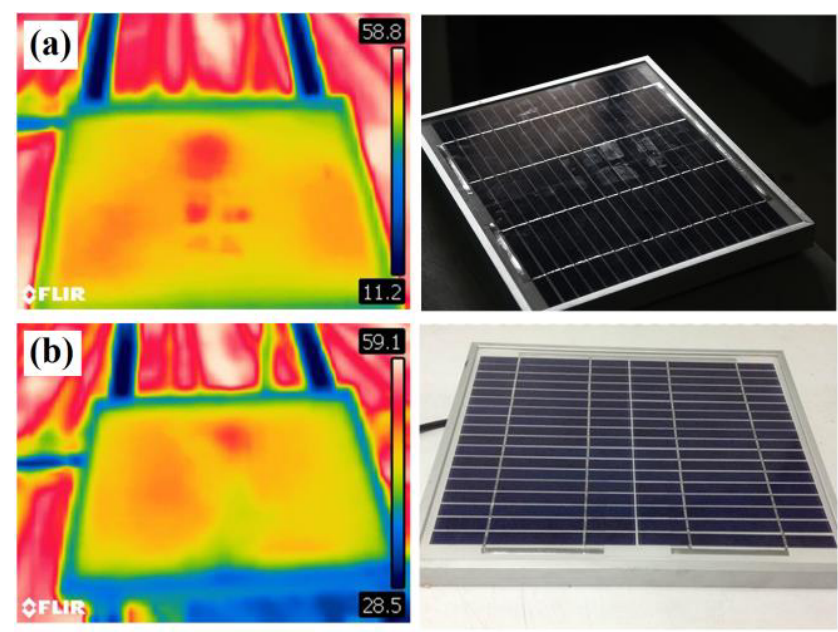

Figure 9. Infrared images and photos of the $10 \mathrm{~W}$ solar PV module taken (a) with the adhesive and (b) without the adhesive.

When the adhesive was removed and infrared images were taken again, only one small hotspot remained on the PV module, shown in Fig. 9(b). This corresponds to the localized heating at the junction box. The hotspot area which initially affected $4.54 \%$ of the module was reduced to $1.61 \%$ when the shading factor was removed. This results support the observations that anything that physically blocks the solar PV module from direct sun exposure, causing a partial shading effect, can lead to hotspots.

\section{Conclusions}

The thermal imaging system developed in this study evaluated different solar PV modules and detected potential hotspots. Through infrared thermography and kmeans clustering, local heating areas were isolated. Relative percent area affected by the hotspot as well as the temperature were obtained using the Hotspot Detection algorithm developed.

There are many factors that contribute to the existence of hotspots on solar PV modules. Among the possible causes of hotspots are underlying defects such as a crack. Junction boxes, found at the back of the PV modules also contribute to hotspots. Even adhesives, which caused partial shading on the solar PV module contributed to hotspot formation. Anything, no matter the size, that directly covers the solar PV module from the sun contributes to shading and can lead to hotspots.

\section{Acknowledgement}

The researchers would like to express their gratitude to the Department of Science and Technology Engineering Research and Development for Technology (DOST-ERDT) for supporting this study, and Ateneo de Davao University School of Engineering and Architecture for supporting A.M. Salazar.

\section{References}

1. Renewables 2015 Global Status Report - Key Findings, 8 (2015) REN21

2. Q Cells, Retrieved from https://www.qcells.com/products/technology.html

3. C. Ibarra-Castanedo, A. Bendada, and X. P. V Maldague, 5th Pan American Conference for NDT, (2011).

4. M. C. Chandhok, S. Chaturvedi, and A A Khurshid, IJIT 111 (2012).

5. Mathworks Retrieved from http://www.mathworks.com/help/stats/k-meansclustering.html\#brah7f_-1.

6. R. Mente, B. Dhandra and G. Mukarambi, IJCSE 3 51 (2014).

7. NPL. Retrived from http://www.npl.co.uk/reference/faqs/what-isemissivity-and-why-is-it-important-(faq-thermal)

8. FLIR. Retrived from http://www.flir.co.uk/instruments/building/display/?i $\mathrm{d}=41872$ 